\title{
The "William Tell" sign: difficult airway resulting from cranial transfixion by an arrow
}

\author{
Mathieu Martin, MD • Roman Mounier, MD • Fabrice Cook, MD • David Lobo, MD • \\ Gilles Dhonneur, MD, PhD
}

Received: 21 January 2016/Revised: 2 February 2016/Accepted: 8 February 2016/Published online: 16 February 2016

(C) Canadian Anesthesiologists' Society 2016

\begin{abstract}
A 53-yr-old man was admitted to our trauma center with a self-inflicted transfixing head injury from an arrow fired from a compressed-air harpoon gun during a suicide attempt. The patient provided written consent for this report. On arrival, the patient was conscious, with a Glasgow coma score of 15 . He could hardly speak, however, as the arrow had transfixed both the pharyngeal and oral tissues to the surrounding bony structures (Figure, left panel). A computed tomography scan of the cranium showed the course of the arrow through the head and neck (Figure, centre panel). The penetration point was slightly lateral (right) and just above the cricoid cartilage. The arrow's pathway continued through the posterior tongue muscles and the soft palate, crossing into the right nasal turbinate (Figure, right panel) and perforating the sphenoid sinus before transiting through the intracranial space. It
\end{abstract}

M. Martin, MD (ه) - R. Mounier, MD · F. Cook, MD .

D. Lobo, MD · G. Dhonneur, MD, PhD

Surgical Intensive Care Unit - Trauma Center, Department of

Anaesthesiology and Critical Care Medicine, Henri Mondor

University Hospital of Paris (APHP), Creteil, France

e-mail: mathieu.martin@hmn.aphp.fr exited medial to the vertex. There was no vascular injury. Urgent neurosurgical removal of the arrow was planned and required securing the airway before general anesthesia could be induced.

Preoperative airway evaluation showed that flexionextension of the neck and opening the mouth were not possible. Midline access to the cricothyroid membrane was also not possible because of the proximity of the arrow. A lateral approach for a tracheostomy was considered-but only if the planned awake flexible bronchoscopic nasotracheal intubation could not be accomplished.

After topicalization of the upper airway with $5 \mathrm{~mL}$ of $5 \%$ aerosolized lidocaine and a low-dose remifentanil infusion (target concentration $<1 \mu \mathrm{g} \cdot \mathrm{mL}^{-1}$ ) to allow both sedation and suctioning blood in the airway, a cuffed 6.5 mm Ruschelit $^{\circledR}$ endotracheal tube (ETT) (Teleflex Medical; Athlone, Ireland) was passed uneventfully into the trachea via the left nostril. Passage was safely completed within ten minutes. The arrow was removed intraoperatively without complication, and the patient was discharged from the hospital one month later with minor visual field abnormalities resulting from the proximity of the arrow to the optic chiasma.

Emergency management of the anticipated difficult airway requires great skill. Among the techniques available, one must give careful consideration to both awake bronchoscopic intubation and direct surgical tracheal access. These two techniques can now be simulated quite simply on realistic manikins. We did make plans for lateral tracheostomy had our awake intubation failed, but the technical difficulty and the patient's anxiety prevented us from attempting that approach first. One concern during our management of this patient was the potentially increased risk of infection 

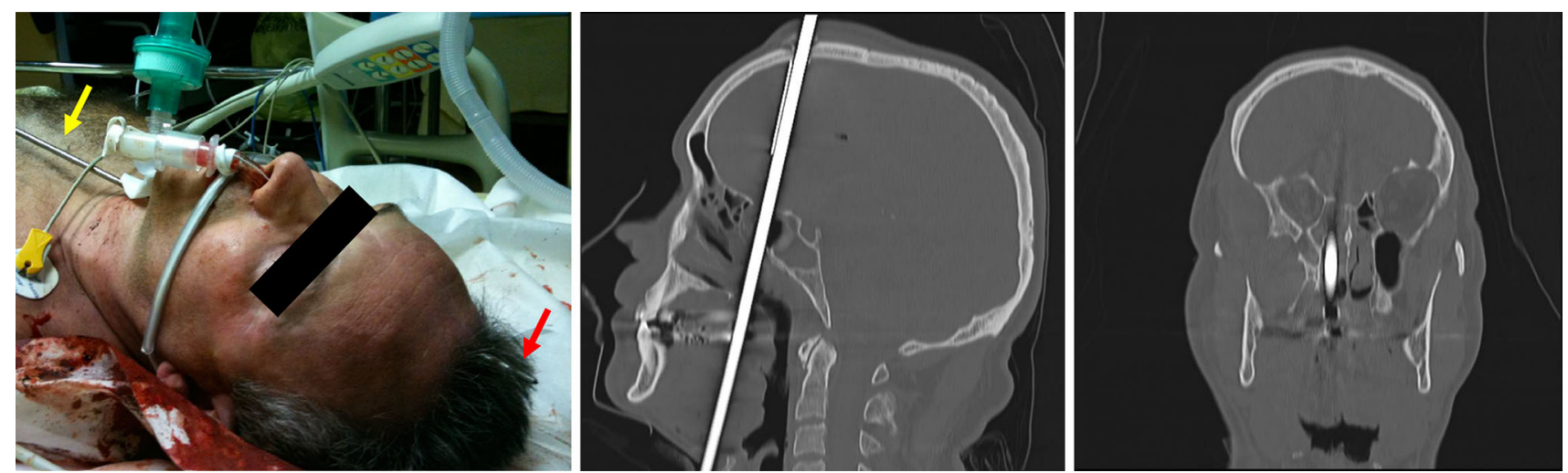

Figure Left panel; the arrow entered just above the cricoid cartilage (yellow arrow) and exited at the top of the cranium (red arrow). Middle panel; the tongue and other pharyngeal structures were transfixed by the arrow to the surrounding bony structures. Right

because of nasotracheal intubation in the setting of a penetrating skull base fracture. Therefore, the patient was given amoxicillin and clavulanic acid as antibiotic prophylaxis (continued for $48 \mathrm{hr}$ ). Also, the ETT was panel; because the arrow travelled through the right nasal passage, awake bronchoscopy-guided nasotracheal intubation was accomplished through the left nares

present for only a brief period of time (i.e., he was extubated shortly after the end of the operation).

Conflicts of interest None declared. 